\title{
NovedADES EN TORNO AL NÚCLEO DE ARTE LEVANTINO DE LA Sierra de Albarracín. El abrigo de Prao Medias (TORMÓN, TERUEL)
}

El abrigo de Prao Medias, descubierto por L. Fortea y R. Canet en 2008, forma parte de un amplio conjunto de estaciones decoradas halladas recientemente en el término de Tormón (Teruel) (fig. 1), siendo la que nos ocupa una de las más destacadas por la temática contenida, grado de conservación de las pinturas, conjunción de motivos con diferentes tonalidades (rojo y blanco) y por la existencia de superposiciones.

El panel decorado se abre hacia el E, siendo de dimensiones medias, contando con $15 \mathrm{~m}$ de longitud y unos $2 \mathrm{~m}$ de altura, con una visera de apenas un metro que protege a las pinturas. El panel se encuentra a unos $20 \mathrm{~m}$ por encima del fondo del barranco, de forma que la visibilidad de éste desde el abrigo resulta un elemento definitorio de primer orden en la elección del emplazamiento, dominando el paso del barranco, en una zona de estrechamiento. Diversos agentes erosivos han afectado de forma notable a la conservación del conjunto pictórico y del propio soporte, con abundantes pérdidas de materia por efecto de la arenización y por el levantamiento por abombamiento de la parte superficial de la roca.

\section{DESCRIPCIÓN DE LOS MOTIVOS}

En el panel se observan un mínimo de dos representaciones de ciervos machos adultos (fig. 2, 1 y 2,2) y una tercera, más dudosa, identificable como los restos de un posible cuadrúpedo de especie indeterminada (fig. 2,3), quizá un équido. Se observan, además, restos de otras dos representaciones no bien definidas, de tonalidad blanquecina, que podrían corresponderse con una figura humana y un objeto indeterminado (fig. $2,4$ y 2,5$)$.

\section{MOTIVO 1}

Ciervo macho adulto orientado a la derecha (fig. 3, 1a). Se aprecia casi la totalidad del cuerpo, aunque la parte delantera (sobre todo cabeza, cuello y astas) se encuentra muy afectada por la erosión. La actitud del animal es estática, aspecto que se evidencia con la disposición vertical y paralela de las patas traseras. Las delanteras adoptan una disposición en "V" invertida, con un ángulo muy agudo. 

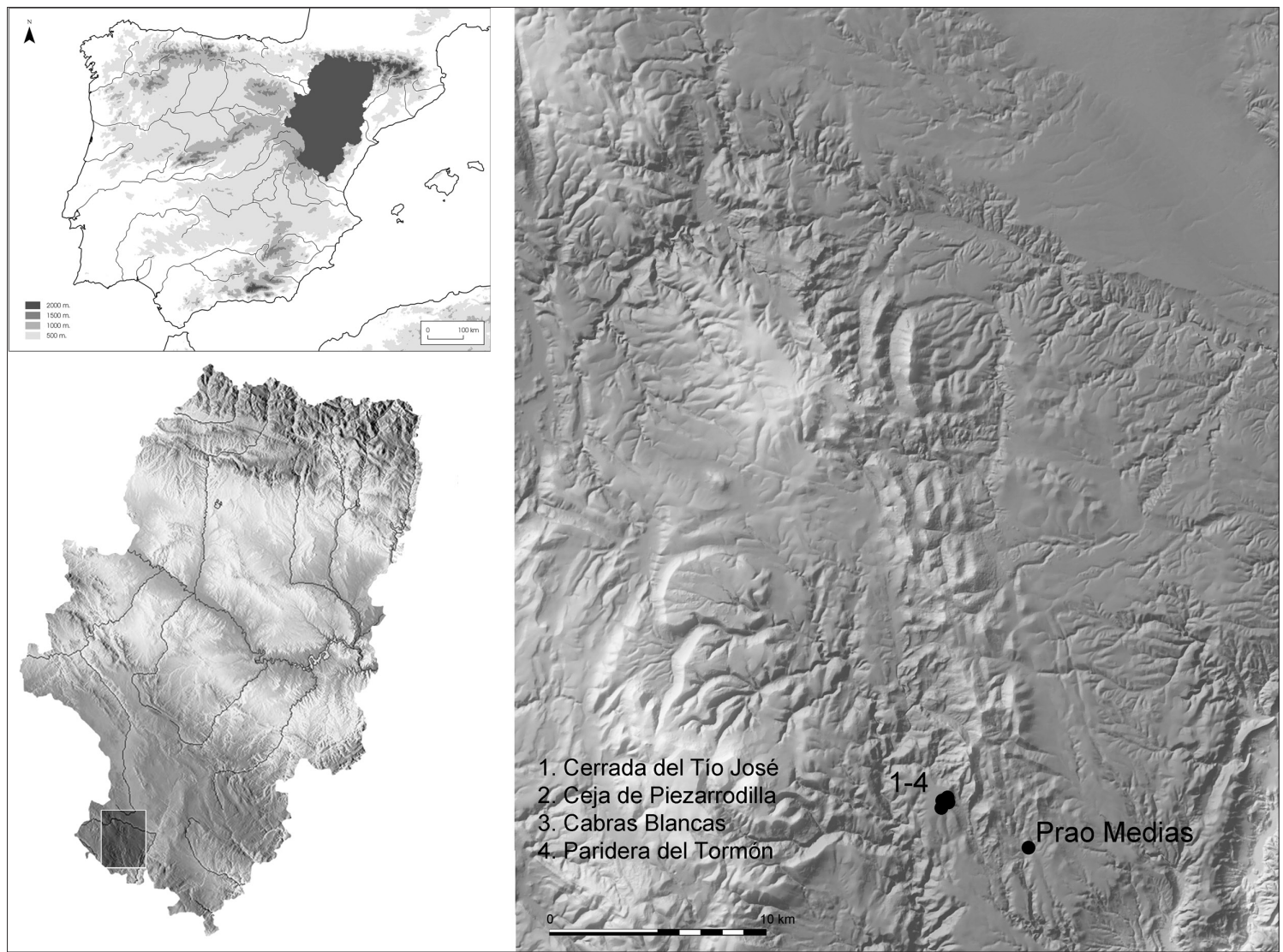

Fig. 1. Mapa de localización del abrigo de Prao Medias en relación con los ya conocidos.

El grado de naturalismo en su representación resulta evidente, con gran limpieza y finura en la confección de las extremidades, en las que se representaron el corvejón y las pezuñas de perfil, aunque en una de las patas traseras parece intuirse el uso de la perspectiva semitorcida, subrayando la naturaleza bisulca de la pezuña.

Se aprecia bien el perfilado del motivo, un trazo relativamente fino que delimita el exterior del ciervo. Sin embargo, y junto a esta delimitación, se observa claramente el añadido externo de un trazo preciso y ancho que determina un módulo más grueso para la figura.

Este segundo perfilado o repintado se aprecia, sobre todo, en la zona superior del cuerpo, cuarto trasero y pecho, sin que se pueda documentar en otras zonas como cuello, cabeza o astas. Si se eliminara el citado repintado el módulo de representación de la figura, mucho más estilizado, aparecería más acorde con el patrón observado en representaciones de cérvidos geográficamente cercanas, como los motivos 10 y 13 del Barranco de las Olivanas.

Se aprecian restos de una especie de trazo vertical en la cruz del animal (motivo 1a), sin que podamos realizar mayores precisiones al respecto.

Dimensiones: $39 \mathrm{~cm}$ longitud máxima, $23 \mathrm{~cm}$ de altura máxima. Color: rojo oscuro.

\section{MOTIVO 2}

Ciervo macho adulto orientado a la derecha (fig. 3, 1b). Se superpone sutilmente al motivo 1. Sólo se conserva la mitad delantera del animal, permitiendo reconocer perfectamente la cabeza, oreja y astas del cérvido. El cuello presenta una tendencia más estilizada que la del motivo anterior, quizá por no presentar el repintado añadido que sí se aprecia en el motivo 1.

La mitad delantera se conserva en relativo buen estado, lo que permite realizar algunas precisiones. Así, se 
documenta el gusto por el realismo mediante la representación del papo y la oreja izquierda, aunque la definición global de la cabeza, atendiendo a la quijada y al morro, carece del detallismo observado en otros cérvidos de la zona ya referidos.

Dimensiones: 20,2 cm de longitud (conservado), 28 $\mathrm{cm}$ de altura máxima. Color: rojo oscuro.

\section{MOTIVO 3}

Posible grupa de un zoomorfo orientado a la derecha (fig. 2, 3). Se localiza a $14,5 \mathrm{~cm}$ por encima del motivo 2. La mala conservación global de la figura hace que sólo se puedan intuir trazos que se corresponderían con la cola y pata trasera del animal. Un gran saltado del soporte afecta directamente a la mitad delantera del motivo, aunque sería posible reconocer restos, quizá, identificables como el pecho y cuello. En la zona superior, se aprecia un resto lineal de tendencia vertical, similar al observado en el ciervo 1 (fig. 2, 1a).

Dimensiones: 17,8 cm de longitud (zona conservada). Color: blanco-amarillento.

\section{MOTIVO 4}

Restos de tonalidad blancuzca cerca del motivo 1, a escasos centímetros por encima y prácticamente yuxtapuestos al asta izquierda del ciervo (fig. 3, 2b). Se podría definir como un elemento de morfología globular, con cierto desarrollo curvilíneo hacia la izquierda. Su identificación resulta problemática, si bien puede recordar tanto a la conformación de los cuartos traseros de un cuadrúpedo como a la típica cabeza con tocados triangulares de gran tamaño (Alonso y Grimal 1999: 122).

Justo por encima de la testuz del ciervo, entre las astas, se observan restos de tonalidad blanquecina que estarían en relación con los anteriormente descritos y que, quizá, pudieran corresponder con parte del desarrollo del cuerpo del motivo propuesto.

Dimensiones: $6,3 \mathrm{~cm}$ de anchura máxima. Color: blanco.

\section{MOTIVO 5}

Otro de los restos observados, a la derecha del anterior y muy próximo a éste, se define como un elemento de tendencia lineal vertical cuyo extremo superior, afectado parcialmente por un desplacado, parece adoptar una forma bidente, de manera que se podría definir como algún tipo de instrumento o como parte de las astas de un ciervo. Resulta importante destacar que este motivo se encuentra infrapuesto a uno de los candiles del asta derecha del ciervo 1 .

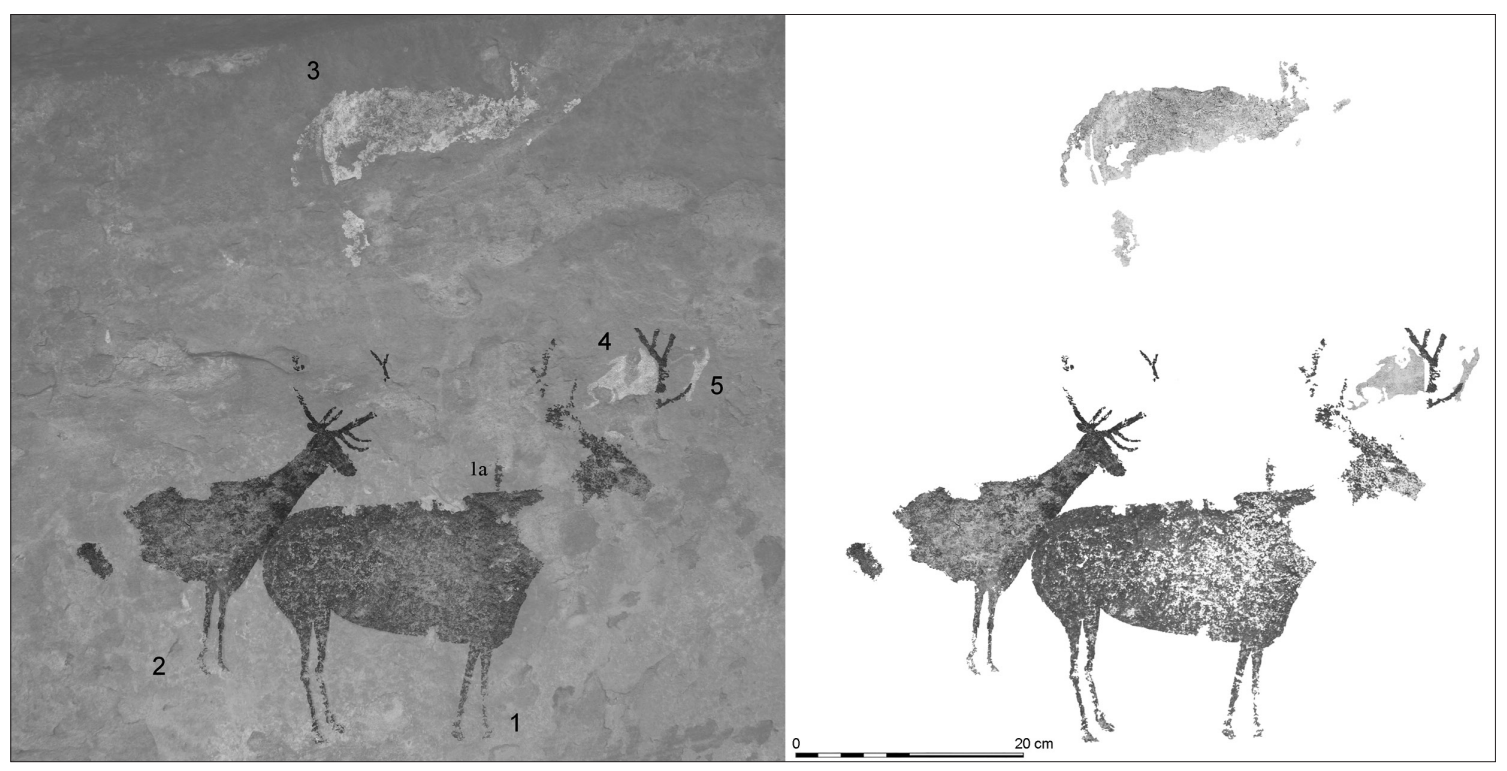

Fig. 2. Calco del panel decorado de Prao Medias. 

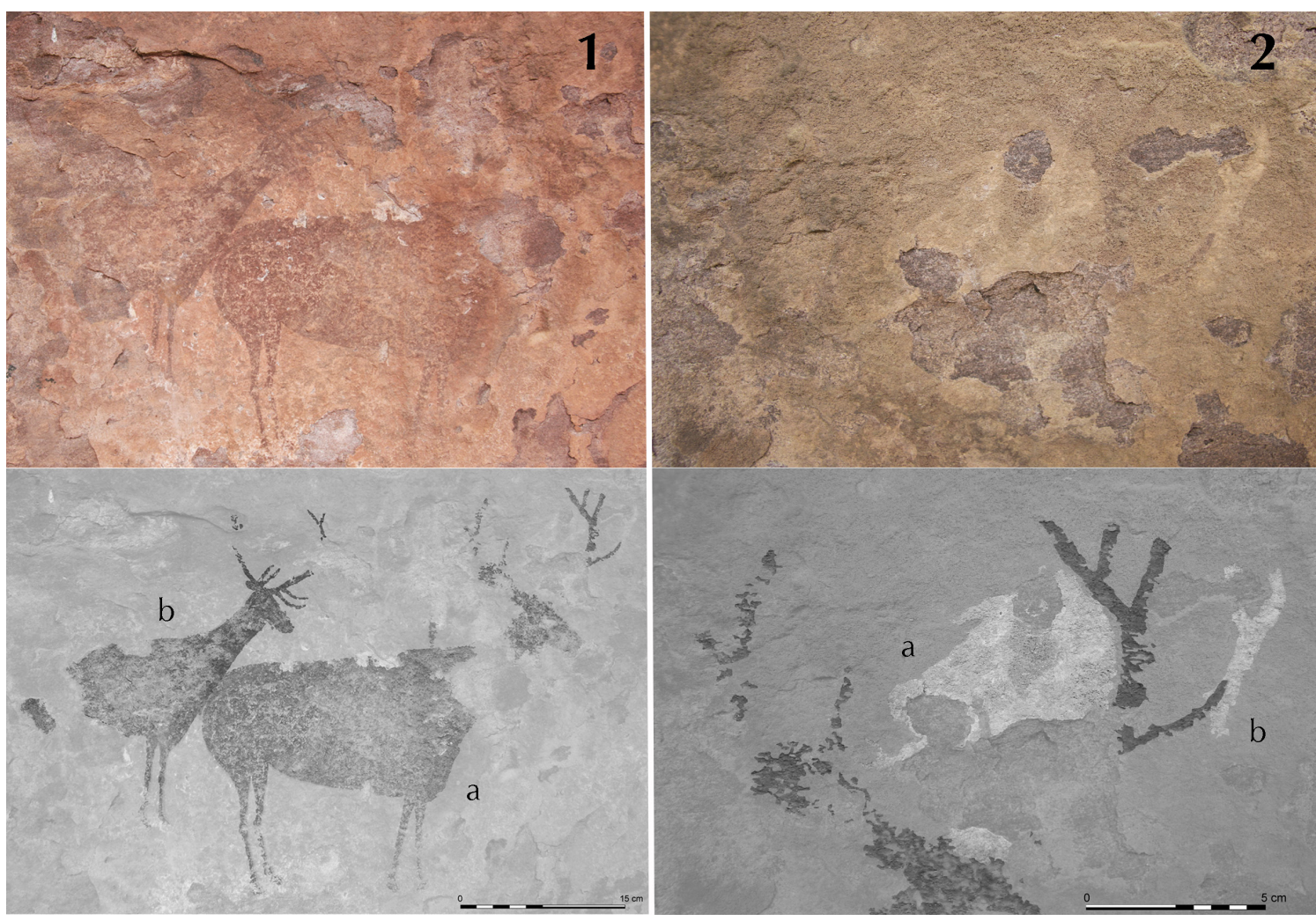

Fig. 3. 1. Detalle de los ciervos (motivos 1 y 2). 2. Detalle de los motivos 4 y 5 .

A favor de su interpretación como un instrumento bidente indeterminado cabe destacar que, en el cercano conjunto de la Paridera del Tormón, se ha definido un antropomorfo de color blanco que porta un instrumento rematado en una horquilla o bidente (Beltrán 1997: 47). Como paralelos también se deben citar los motivos definidos como antropomorfos esquemáticos del abrigo de las Figuras Amarillas (Albarracín), todos ellos elementos lineales con un extremo bidente, aunque en uno de los casos la parte inferior se ramifica en cuatro apéndices (Piñón 1982: 93).

Dimensiones: 4,9 cm de altura máxima; $0,57 \mathrm{~cm}$ de grosor del trazo. Color: blanco.

\section{CONCLUSIONES}

La importancia del conjunto resulta manifiesta, por una parte, si atendemos al realismo de sus figuraciones, que las aleja conceptualmente del conjunto mayoritario de figuraciones zoomorfas de la Sierra de Albarracín. Estas representaciones de grandes y majestuosos ciervos machos, presentes en el arte levantino ya sea aislados o en pareja en casi toda su distribución geográfica (Viñas y Saucedo 2000), aparecerían más próximas a conjuntos de la zona nororiental del arte levantino, sobre todo del Maestrazgo turolense-castellonense y de la cuenca del Matarraña.

Junto a esto, la existencia de superposiciones abre un campo interpretativo y de ordenación cronológica relativa de gran importancia. Resulta evidente la existencia de, al menos, dos fases decorativas diferenciadas en el conjunto de Prao Medias. Una primera en la que serían realizadas las representaciones de color blanco. Una segunda con la figuración de los ciervos, patente en la clara superposición de uno de los candiles de las astas del motivo 1 sobre el 5, apreciándose también la perfecta adaptación del desarrollo del asta a la morfología curvilínea del motivo 4, sobre el que no llega a superponerse. Se documenta una segunda superposición en el abrigo, la ya apuntada entre el ciervo 2 sobre el 1. Sin embargo, resulta interesante subrayar que esta superposición se realiza, a su vez, sobre un repintado previo realizado en toda la línea dorsal, 
grupa y pecho del ciervo 1. Dicho repintado, ligeramente más oscuro y de textura más densa que lo apreciado en el resto de la figura, parece iniciarse justo en el punto donde se documenta un elemento de tendencia lineal y vertical externo al ciervo (motivo 1a).

Así, atendiendo al caso que nos ocupa, las representaciones de animales de grandes dimensiones, estáticas y de color rojo se realizaron con posterioridad a los restos de coloración blanquecina.

La Sierra de Albarracín cuenta con temas y rasgos estilísticos clásicos dentro del arte levantino. Sin embargo, las múltiples singularidades artísticas que presenta este núcleo parecen diluir los patrones clásicos levantinos, siendo éstos ciertamente minoritarios, bien por una peor conservación o bien por una efectiva menor presencia cuantitativa.

La Sierra de Albarracín, con los sub-núcleos de Albarracín, Bezas y Tormón, aparece como un territorio bisagra en el que se documentan tradiciones artísticas (ciclos) de tendencia naturalista que no tienen porqué formar parte de un mismo y único ciclo levantino (Berrocal 2005; Martínez Bea 2005 y 2008). Nuevos conjuntos rupestres descubiertos, como el de Prao Medias, aportarán una información que estimamos determinante para la comprensión del fenómeno rupestre levantino, no sólo en la Sierra de Albarracín sino en el conjunto de la península Ibérica, y para el que, probablemente, se deba plantear una redefinición, al menos, terminológica.

\author{
Manuel Bea Martínez \\ Investigador postdoctoral "Torres Quevedo" \\ MIMECO \& 3D Scanner \\ Spinn-off UZ Grupo PPVE \\ Universidad de Zaragoza \\ manubea@unizar.es
}

\section{BIBLIOGRAFÍA}

ALONSO, A.; GRIMAL, A. (1999): Introducción al Arte Levantino a través de una estación singular: La Cueva de la Vieja (Alpera, Albacete), Albacete.

BELTRÁN, A. (1997): Las pinturas prehistóricas de la Paridera de Tormón, Arqueología Aragonesa 1994, 45-49.

BERROCAL, M. C. (2005): Paisaje y arte rupestre. Patrones de localización de la pintura levantina, BAR International Series 1409, Oxford.

MARTÍNEZ-BEA, M. (2005): Variabilidad estilística y distribución espacial del arte rupestre levantino en Aragón: el ejemplo de La Vadaa (Castellote, Teruel), Zaragoza.

MARTÍNEZ-BEA, M. (2008): Arte rupestre de Albarracín: la excepcionalidad de un conjunto interior, Actas del IV Congreso de Neolítico Peninsular (M. S. Hernández, J. A. Soler, J. A. López, eds.), Alicante, 141-148.

PIÑÓN, F. (1982): Las pinturas rupestres de Albarracín (Teruel), Madrid.

VIÑAS, R.; SAUCEDO, E. (2000): Los cérvidos en el arte rupestre postpaleolítico, $Q P A C$ 21, 53-68. 